\title{
Vertex-Coloring Edge-Weighting of Bipartite Graphs with Two Edge Weights*
}

\author{
Hongliang Lud
}

School of Mathematics and Statistics, Xi'an Jiaotong University, Xi'an, PR China

received $28^{\text {th }}$ Feb. 2015, revised $29^{\text {th }}$ Oct. 2015, accepted $9^{\text {th }}$ Dec. 2015.

Let $G$ be a graph and $\mathcal{S}$ be a subset of $Z$. A vertex-coloring $\mathcal{S}$-edge-weighting of $G$ is an assignment of weights by the elements of $\mathcal{S}$ to each edge of $G$ so that adjacent vertices have different sums of incident edges weights.

It was proved that every 3 -connected bipartite graph admits a vertex-coloring $\mathcal{S}$-edge-weighting for $\mathcal{S}=\{1,2\}$ (H. Lu, Q. Yu and C. Zhang, Vertex-coloring 2-edge-weighting of graphs, European J. Combin., 32 (2011), 22-27). In this paper, we show that every 2 -connected and 3-edge-connected bipartite graph admits a vertex-coloring $\mathcal{S}$-edgeweighting for $\mathcal{S} \in\{\{0,1\},\{1,2\}\}$. These bounds we obtain are tight, since there exists a family of infinite bipartite graphs which are 2-connected and do not admit vertex-coloring $\mathcal{S}$-edge-weightings for $\mathcal{S} \in\{\{0,1\},\{1,2\}\}$.

Keywords: edge-weighting, vertex-coloring, 2-connected, bipartite graph

\section{Introduction}

In this paper, we consider only finite, undirected and simple connected graphs. For a vertex $v$ of graph $G=(V, E), N_{G}(v)$ denotes the set of vertices which are adjacent to $v$ and $d_{G}(v)=\left|N_{G}(v)\right|$ is called the degree of vertex $v$. Let $\delta(G)$ and $\triangle(G)$ denote the minimum degree and maximum degree of graph $G$, respectively. For $v \in V(G)$ and $r \in Z^{+}$, let $N_{G}^{r}(v)=\left\{u \in N(v) \mid d_{G}(u)=r\right\}$. If $v \in V(G)$ and $e \in E(G)$, we use $v \sim e$ to denote that $v$ is an end-vertex of $e$. For two disjoint subsets $S, T$ of $V(G)$, let $E_{G}(S, T)$ denote the subset of edges of $E(G)$ with one end in $S$ and other end in $T$ and let $e_{G}(S, T)=\left|E_{G}(S, T)\right|$. Let $G=(U, W, E)$ denote a bipartite graph with bipartition $(U, W)$ and edge set $E$.

Let $\mathcal{S}$ be a subset of $Z$. An $\mathcal{S}$-edge-weighting of a graph $G$ is an assignment $w: E(G) \rightarrow S$. An $\mathcal{S}$ edge-weighting $w$ of a graph $G$ induces a coloring of the vertices of $G$, where the color of vertex $v$, denoted by $c(v)$, is $\sum_{e \sim v} w(e)$. An $\mathcal{S}$-edge-weighting of a graph $G$ is a vertex-coloring if for every edge $e=u v$, $c(u) \neq c(v)$ and we say that $G$ admits a vertex-coloring $\mathcal{S}$-edge-weighting. If $\mathcal{S}=\{1,2, \ldots, k\}$, then a vertex-coloring $\mathcal{S}$-edge-weighting of a graph $G$ is usually called a vertex-coloring $k$-edge-weighting.

For vertex-coloring edge-weighting, Karoński et al. (2004) posed the following conjecture:

\footnotetext{
*This work was supported by the National Natural Science Foundation of China No. 11471257 and the Fundamental Research Funds for the Central Universities.

†Corresponding email: luhongliang215@sina.com

1365-8050 @ 2015 Discrete Mathematics and Theoretical Computer Science (DMTCS), Nancy, France
} 
Conjecture 1.1 Every graph without isolated edges admits a vertex-coloring 3-edge-weighting.

This conjecture is still wide open. Karoński et al.(2004) showed that Conjecture 1.1 is true for 3-colorable graphs. Recently, Kalkowski et al. (2010) showed that every graph without isolated edges admits a vertexcoloring 5-edge-weighting. This result is an improvement on the previous bounds on $k$ established by Addario-Berry et al. (2007), Addario-Berry et al. (2008), and Wang and Yu (2008), who obtained the bounds $k=30, k=16$, and $k=13$, respectively.

Many graphs actually admit a vertex-coloring 2-edge-weighting (in fact, experiments suggest (see Addario-Berry et al. (2008) ) that almost all graphs admit a vertex-coloring 2-edge-weighting), however it is not known which ones do not. Khatirinejad et al. (2012) explored the problem of classifying those graphs which admit a vertex-coloring 2-edge-weighting. Chang et al. (2011) had made some progress in determining which classes of graphs admit vertex-coloring 2-edge-weighting, and proved that there exists a family of infinite bipartite graphs (e.g., the generalized $\theta$-graphs) which are 2-connected and admit a vertex-coloring 3 -edge-weighting but not vertex-coloring 2-edge-weightings. Lu et al. (2011) showed that every 3 -connected bipartite graph admits a vertex-coloring 2-edge-weighting.

We write

$$
\begin{aligned}
& \mathscr{G}_{12}=\{G \mid G \text { admits a vertex-coloring }\{1,2\} \text {-edge-weighting }\} ; \\
& \mathscr{G}_{01}=\{G \mid G \text { admits a vertex-coloring }\{0,1\} \text {-edge-weighting }\} ; \\
& \mathscr{G}_{12}^{*}=\{G \mid G \text { is bipartite and admits a vertex-coloring }\{1,2\} \text {-edge-weighting }\} ; \\
& \mathscr{G}_{01}^{*}=\{G \mid G \text { is bipartite and admits a vertex-coloring }\{0,1\} \text {-edge-weighting }\} .
\end{aligned}
$$

Dudek and Wajc (2011) showed that determining whether a graph belongs to $\mathscr{G}_{12}$ or $\mathscr{G}_{01}$ is NP-complete. Moreover, they showed that $\mathscr{G}_{12} \neq \mathscr{G}_{01}$. The counterexamples constructed by Dudek and Wajc (2011) are non-bipartite.

Now we construct a bipartite graph, which admits a vertex-coloring 2-edge-weighting but not vertexcoloring $\{0,1\}$-edge-weightings. Let $C_{6}$ be a cycle of length six and $\Gamma$ be a graph obtained by connecting an isolated vertex to one of the vertices of $C_{6}$. Take two disjoint copies of $\Gamma$. Connect two vertices of degree one of the two copies and this gives a connected bipartite graph $G$. It is easy to prove that $G$ admits a vertex-coloring 2 -edge-weighting but not vertex-coloring $\{0,1\}$-edge-weighting. Hence $\mathscr{G}_{01}^{*} \neq \mathscr{G}_{12}^{*}$. Next we would like to propose the following problem.

Problem 1 Determining whether a graph $G \in \mathscr{G}_{12}^{*}$ or $G \in \mathscr{G}_{01}^{*}$ is polynomial?

In this paper, we characterize bipartite graphs which admit a vertex-coloring $\mathcal{S}$-edge-weighting for $\mathcal{S} \in\{\{0,1\},\{1,2\}\}$, and obtain the following result.

Theorem 1.2 Let $G$ be a 3-edge-connected bipartite graph $G=(U, W, E)$ with minimum degree $\delta(G)$. If $G$ contains a vertex $u$ of degree $\delta(G)$ such that $G-u$ is connected, then $G$ admits a vertex-coloring $\mathcal{S}$-edge-weighting for $\mathcal{S} \in\{\{0,1\},\{1,2\}\}$.

By Theorem 1.2. it is easy to obtain the following result, which improves and extends the result obtained by Lu et al. (2011).

Theorem 1.3 Every 2-connected and 3-edge-connected bipartite graph $G=(U, W, E)$ admits a vertexcoloring $\mathcal{S}$-edge-weighting for $\mathcal{S} \in\{\{0,1\},\{1,2\}\}$. 
So far, all known counterexamples of bipartite graphs, which do not have vertex-coloring $\{0,1\}$-edgeweightings or vertex-coloring $\{1,2\}$-edge-weightings are graphs with minimum degree 2 . So we would like to propose the following problem.

Problem 2 Does every bipartite graph with $\delta(G) \geq 3$ admit a vertex-coloring $\mathcal{S}$-edge-weighting, where $\mathcal{S} \in\{\{0,1\},\{1,2\}\}$.

A factor of a graph $G$ is a spanning subgraph. For a graph $G$, there is a close relationship between 2-edge-weighting and graph factors. Namely, a 2-edge-weighting problem is equivalent to finding special factors of graphs (see Addario-Berry et al. (2007) and Addario-Berry et al. (2008)). So to find factors with pre-specified degree is an important part of edge-weighting.

Let $g, f: V(G) \rightarrow Z$ be two integer-valued functions such that $g(v) \leq f(v)$ and $g(v) \equiv f(v)$ $(\bmod 2)$ for all $v \in V(G)$. A factor $F$ of $G$ is called $(g, f)$-parity factor if $g(v) \leq d_{F}(v) \leq f(v)$ and $d_{F}(v) \equiv f(v)(\bmod 2)$ for all $v \in V(G)$. For $X \subseteq V(G)$, we write $g(X)=\sum_{x \in X} g(x)$ and $f(X)$ is defined similarly. For $(g, f)$-parity factors, Lovász obtained a sufficient and necessary condition.

Theorem 1.4 (Lovász (1972)) A graph $G$ contains a $(g, f)$-parity factor if and only if for any two disjoint subsets $S$ and $T$ of $V(G)$, it follows that

$$
\eta(S, T)=f(S)-g(T)+\sum_{x \in T} d_{G-S}(x)-\tau(S, T) \geq 0,
$$

where $\tau(S, T)$ denotes the number of components $C$, called g-odd components of $G-S-T$ such that $g(V(C))+e_{G}(V(C), T) \equiv 1(\bmod 2)$.

In the proof of the main theorems, we also need the following three lemmas.

Theorem 1.5 (Chang et al. (2011)) Every non-trivial connected bipartite graph $G=(A, B, E)$ with $|A|$ even, admits a vertex-coloring 2-edge-weighting $w$ such that $c(u)$ is odd for $u \in A$ and $c(v)$ is even for $v \in B$.

Theorem 1.6 (Chang et al. (2011)) Let $r \geq 3$ be an integer. Every $r$-regular bipartite graph $G$ admits a vertex-coloring 2-edge-weighting.

Theorem 1.7 (Khatirinejad et al. (2012)) Every $r$-regular graph $G$ admits a vertex-coloring 2-edgeweighting if and only if it admits a vertex-coloring $\{0,1\}$-edge-weighting.

\section{Proof of Theorem 1.2}

Corollary 2.1 Every non-trivial connected bipartite graph $G=(A, B, E)$ with $|A|$ even admits a vertexcoloring $\{0,1\}$-edge-weighting.

Proof: By Theorem 1.5 $G$ admits a vertex-coloring 2-edge-weighting $w$ such that $c(u)$ is odd for $u \in A$ and $c(v)$ is even for $v \in B$. Let $w^{\prime}(e)=0$ if $w(e)=2$ and $w^{\prime}(e)=1$ if $w(e)=1$. Then $w^{\prime}$ is a vertex-coloring $\{0,1\}$-edge-weighting of graph $G$.

For completing the proof of Theorem 1.2 , we need the following two technical lemmas. 
Lemma 2.2 Let $G$ be a bipartite graph with bipartition $(U, W)$, where $|U| \equiv|W| \equiv 1(\bmod 2)$. Let $\delta(G)=\delta$ and $u \in U$ such that $d_{G}(u)=\delta$. If one of the following two conditions holds, then $G$ contains a factor $F$ such that $d_{F}(u)=\delta, d_{F}(x) \equiv \delta+1(\bmod 2)$ for all $x \in U-u, d_{F}(y) \equiv \delta(\bmod 2)$ for all $y \in W$ and $d_{F}(y) \leq \delta-2$ for all $y \in N_{G}^{\delta}(u)$.

(i) $\delta(G) \geq 4, G$ is 3-edge-connected and $G-u$ is connected.

(ii) $\delta(G)=3, G$ is 3-edge-connected and $\left|N_{G}^{\delta}(u)\right| \leq 2$.

Proof: Let $M$ be an integer such that $M \geq \triangle(G)$ and $M \equiv \delta(\bmod 2)$. Let $m \in\{0,-1\}$ such that $m \equiv \delta(\bmod 2)$. Let $g, f: V(G) \rightarrow Z$ such that

$$
g(x)= \begin{cases}\delta & \text { if } x=u, \\ m-1 & \text { if } x \in U-u, \\ m & \text { if } x \in W\end{cases}
$$

and

$$
f(x)= \begin{cases}M+1 & \text { if } x \in U-u, \\ M & \text { if } x \in(W \cup\{u\})-N_{G}^{\delta}(u), \\ \delta-2 & \text { if } x \in N_{G}^{\delta}(u) .\end{cases}
$$

By definition, we have $g(v) \equiv f(v)(\bmod 2)$ for all $v \in V(G)$. It is sufficient for us to show that $G$ contains a $(g, f)$-parity factor. Indirectly, suppose that $G$ contains no $(g, f)$-parity factors. By Theorem 1.4, there exist two disjoint subsets $S$ and $T$ such that

$$
\eta(S, T)=f(S)-g(T)+\sum_{x \in T} d_{G-S}(x)-\tau(S, T)<0,
$$

where $\tau(S, T)$ denotes the number of $g$-odd components of $G-S-T$. Since $f(V(G))$ is even, by parity, we have

$$
\eta(S, T)=f(S)-g(T)+\sum_{x \in T} d_{G-S}(x)-\tau(S, T) \leq-2
$$

Hence $S \cup T \neq \emptyset$. We choose $S$ and $T$ such that $S \cup T$ is minimal. Let $A=V(G)-S-T$.

Claim 1. $T \subseteq\{u\}$. 
Otherwise, let $v \in T-u$ and $T^{\prime}=T-v$. We have

$$
\begin{aligned}
\eta\left(S, T^{\prime}\right) & =f(S)-g\left(T^{\prime}\right)+\sum_{x \in T^{\prime}} d_{G-S}(x)-\tau\left(S, T^{\prime}\right) \\
& =f(S)-(g(T)-g(v))+\left(\sum_{x \in T} d_{G-S}(x)-d_{G-S}(v)\right)-\tau\left(S, T^{\prime}\right) \\
& \leq f(S)-g(T)+\left(\sum_{x \in T} d_{G-S}(x)-d_{G-S}(v)\right)-\left(\tau(S, T)-e_{G}(v, A)\right)+g(v) \\
& \leq f(S)-g(T)+\sum_{x \in T} d_{G-S}(x)-\tau(S, T)+\left(g(v)-d_{G-S}(v)+e_{G}(v, A)\right) \\
& =\eta(S, T)+\left(g(v)-d_{G-S}(v)+e_{G}(v, A)\right) \\
& \leq \eta(S, T)-\left(d_{G-S}(v)-e_{G}(v, A)\right) \\
& \leq \eta(S, T) \leq-2,
\end{aligned}
$$

contradicting the choice of $S$ and $T$.

Claim 2. $S \subseteq N_{G}^{\delta}(u)$.

Otherwise, suppose that $S-N_{G}^{\delta}(u) \neq \emptyset$ and let $v \in S-N_{G}^{\delta}(u)$. Let $S^{\prime}=S-v$. We have

$$
\begin{aligned}
\eta\left(S^{\prime}, T\right) & =f\left(S^{\prime}\right)-g(T)+\sum_{x \in T} d_{G-S^{\prime}}(x)-\tau\left(S^{\prime}, T\right) \\
& =(f(S)-f(v))-g(T)+\left(\sum_{x \in T} d_{G-S}(x)+e_{G}(v, T)\right)-\tau\left(S^{\prime}, T\right) \\
& \leq f(S)-g(T)+\sum_{x \in T} d_{G-S}(x)-\left(\tau(S, T)-e_{G}(v, A)\right)-f(v)+e_{G}(v, T) \\
& =f(S)-g(T)+\sum_{x \in T} d_{G-S}(x)-\tau(S, T)+\left(e_{G}(v, T)+e_{G}(v, A)-f(v)\right) \\
& \leq \eta(S, T)+\left(d_{G}(v)-f(v)\right) \\
& \leq \eta(S, T) \leq-2,
\end{aligned}
$$

contradicting the choice of $S$ and $T$ again.

We write $\tau(S, T)=\tau$. By Claims 1 and 2, we have

$$
\begin{aligned}
\eta(S, T) & =f(S)-g(T)+\sum_{x \in T} d_{G-S}(x)-\tau \\
& =(\delta-2)|S|-\delta|T|+|T|(\delta-|S|)-\tau \quad \text { (by Claims 1 and 2) } \\
& =(\delta-2-|T|)|S|-\tau \quad \text { (by Claim 1) } \\
& \leq-2
\end{aligned}
$$


i.e.,

$$
(\delta-2-|T|)|S|+2 \leq \tau,
$$

which implies $\tau \geq 2$ since $|T| \leq 1$.

Since $G-u$ is connected, we may see that $S \neq \emptyset$. Note that $G$ is 3-edge-connected, by Claims 1 and 2, we have

$$
\begin{aligned}
3 \tau & \leq e_{G}(A, S \cup T) \\
& =e_{G}(A, S)+e_{G}(A, T) \\
& \leq(\delta-|T|)|S|+|T|(\delta-|S|) \quad \text { (by Claims 1 and 2) } \\
& =(\delta-2|T|)|S|+|T| \delta,
\end{aligned}
$$

i.e.,

$$
3 \tau \leq(\delta-2|T|)|S|+|T| \delta
$$

Combining (2) and (3), we may see that

$$
\delta|T| \geq(2 \delta-|T|-6)|S|+6 .
$$

If $\delta \geq 4$, then we have

$$
\begin{aligned}
\delta & \geq \delta|T| \quad(\text { since }|T| \leq 1) \\
& \geq(2 \delta-|T|-6)|S|+6 \quad \text { (since }|S| \geq 1) \\
& \geq 2 \delta-|T| \\
& \geq 2 \delta-1,
\end{aligned}
$$

a contradiction. So we may assume that $\delta=3$. Note that $|S| \leq\left|N_{G}^{\delta}(u)\right| \leq 2$. By [4], we have

$$
3=\delta \geq \delta|T| \geq-|T||S|+6 \geq 4,
$$

a contradiction again.

This completes the proof.

Lemma 2.3 Let $G$ be a bipartite graph with bipartition $(U, W)$, where $|U| \equiv|W| \equiv 1(\bmod 2)$. Let $\delta(G)=\delta$ and $u \in U$ such that $d_{G}(u)=\delta$. If one of the following two conditions holds, then $G$ contains a factor $F$ such that $d_{F}(u)=0, d_{F}(x) \equiv 1(\bmod 2)$ for all $x \in U-u, d_{F}(y) \equiv 0(\bmod 2)$ for all $x \in W$ and $d_{F}(y) \geq 2$ for all $y \in N_{G}(u)$.

(i) $\delta(G) \geq 4, G$ is 3-edge-connected and $G-u$ is connected.

(ii) $\delta(G)=3, G$ is 3-edge-connected and there exists a vertex $v \in N_{G}(u)$ such that $d_{G}(v)>3$.

Proof: Let $M$ be an even integer such that $M \geq \triangle(G)$. Let $g, f: V(G) \rightarrow Z$ such that

$$
g(x)= \begin{cases}0 & \text { if } x \in(\{u\} \cup W)-N_{G}(u), \\ 2 & \text { if } x \in N_{G}(u), \\ -1 & \text { if } x \in U-u\end{cases}
$$


and

$$
f(x)= \begin{cases}M & \text { if } x \in W \\ 0 & \text { if } x=u, \\ M+1 & \text { if } x \in U-u .\end{cases}
$$

Clearly, $g(v) \equiv f(v)(\bmod 2)$ for all $v \in V(G)$ and $g(V(G))$ is even. It is also sufficient for us to show that $G$ contains a $(g, f)$-parity factor.

Indirectly, suppose that $G$ contains no $(g, f)$-parity factors. By Theorem 1.4 there exist two disjoint subsets $S$ and $T$ such that

$$
\eta(S, T)=f(S)-g(T)+\sum_{x \in T} d_{G-S}(x)-\tau(S, T) \leq-2
$$

where $\tau(S, T)$ denotes the number of $g$-odd components of $G-S-T$. We choose $S$ and $T$ such that $S \cup T$ is minimal. Let $A=V(G)-S-T$.

Claim 1. $S \subseteq\{u\}$.

Otherwise, suppose that there exists a vertex $v \in S-u$. Let $S^{\prime}=S-v$. Then we have

$$
\begin{aligned}
\eta\left(S^{\prime}, T\right) & =f\left(S^{\prime}\right)-g(T)+\sum_{y \in T} d_{G-S^{\prime}}(y)-\tau\left(S^{\prime}, T\right) \\
& =(f(S)-f(v))-g(T)+\left(\sum_{y \in T} d_{G-S}(y)+e_{G}(v, T)\right)-\tau\left(S^{\prime}, T\right) \\
& \leq f(S)-g(T)+\sum_{y \in T} d_{G-S}(y)-f(v)+e_{G}(v, T)-\left(\tau(S, T)-e_{G}(v, A)\right) \\
& =\eta(S, T)-\left(f(v)-e_{G}(v, T)-e_{G}(v, A)\right) \\
& \leq \eta(S, T)-\left(f(v)-d_{G}(v)\right) \\
& \leq \eta(S, T) \leq-2
\end{aligned}
$$

contradicting the the choice of $S \cup T$.

Claim 2. $T \subseteq N_{G}(u)$. 
Otherwise, suppose that $T-N_{G}(u) \neq \emptyset$. Let $x \in T-N_{G}(u)$ and let $T^{\prime}=T-x$. Then we have

$$
\begin{aligned}
\eta\left(S, T^{\prime}\right) & =f(S)-g\left(T^{\prime}\right)+\sum_{y \in T^{\prime}} d_{G-S}(y)-\tau\left(S, T^{\prime}\right) \\
& =f(S)-(g(T)-g(x))+\left(\sum_{y \in T} d_{G-S}(y)-d_{G-S}(x)\right)-\tau\left(S, T^{\prime}\right) \\
& \leq f(S)-(g(T)-g(x))+\left(\sum_{y \in T} d_{G-S}(y)-d_{G-S}(x)\right)-\left(\tau(S, T)-e_{G}(x, A)\right) \\
& =f(S)-g(T)+\sum_{y \in T} d_{G-S}(y)-\tau(S, T)-\left(d_{G-S}(x)-e_{G}(x, A)\right)+g(x) \\
& =\eta(S, T)-\left(d_{G-S}(x)-e_{G}(x, A)\right)+g(x) \\
& \leq \eta(S, T) \leq-2,
\end{aligned}
$$

contradicting the choice of $S \cup T$.

By Claims 1 and 2, we may see that $f(S)=0$ and $g(T)=2|T|$. For simplicity, we write $\tau(S, T)=\tau$. By $\sqrt{6}$, we see that

$$
\tau \geq \sum_{x \in T}\left(d_{G}(x)-|S|\right)-2|T|+2,
$$

which implies

$$
\tau \geq \sum_{x \in T}(\delta-1)-2|T|+2 \geq 2 .
$$

Note that $G-u$ is connected, so we have $|T| \geq 1$. Since $G$ is 3-edge-connected, we have

$$
\begin{aligned}
3 \tau & \leq \sum_{x \in T}\left(d_{G}(x)-|S|\right)+(\delta-|T|)|S| \\
& =\sum_{x \in T} d_{G}(x)+(\delta-2|T|)|S|
\end{aligned}
$$

i.e.,

$$
3 \tau \leq \sum_{x \in T} d_{G}(x)+(\delta-2|T|)|S|
$$

Inequalities (7) and (9) implies

$$
\begin{aligned}
2 \sum_{x \in T} d_{G}(x)+6 & \leq|S||T|+6|T|+\delta|S| \quad \text { (since }|S| \leq 1 \text { and }|T| \geq 1 \text { ) } \\
& \leq 7|T|+\delta
\end{aligned}
$$


i.e.,

$$
7|T| \geq 2 \sum_{x \in T} d_{G}(x)+6-\delta .
$$

If $\delta \geq 4$, by $[10$, , it follows

$$
7|T| \geq 6+\delta(2|T|-1) \geq 8|T|+2,
$$

a contradiction. So we may assume that $\delta=3$. By condition (ii), $\sum_{x \in T} d_{G}(x) \geq 3|T|+1$. Combining (10),

$$
\begin{aligned}
7|T| & \geq 2 \sum_{x \in T} d_{G}(x)+6-\delta \\
& \geq 2(3|T|+1)+3
\end{aligned}
$$

which implies $|T| \geq 5$, a contradiction since $|T| \leq\left|N_{G}(u)\right| \leq 3$.

This completes the proof.

Proof of Theorem 1.2 By Theorem 1.5 and Corollary 2.1, we can assume that both $|A|$ and $|B|$ are odd.

Firstly, we consider $\mathcal{S}=\{0,1\}$. If $G$ is 3-regular, by Theorem 1.6, then $G$ admits a vertex-coloring 2-edge-weighting. By Theorem 1.7, $G$ also admits a vertex-coloring $\{0,1\}$-edge-weighting. So we can assume that $\delta(G) \geq 3$ and $G$ is not 3-regular. If $\delta(G)=3$, since $G$ is 3-edge-connected, then $G-x$ is connected for every vertex $x$ of $G$ with degree three. Hence there exists a vertex $v$ with degree three such that $N_{G}(v)$ contains a vertex with degree at least four. Let

$$
u^{*}= \begin{cases}u & \text { if } \delta \geq 4, \\ v & \text { if } \delta=3 .\end{cases}
$$

Without loss generality, we may assume that $u^{*} \in U$ and so it is a vertex satisfying the conditions of Lemma 2.3. Hence by Lemma 2.3. $G$ contains a factor $F$, which satisfies the following three conditions.

(i) $d_{F}\left(u^{*}\right)=0$;

(ii) $d_{F}(x) \equiv 1(\bmod 2)$ for all $x \in U-u^{*}$;

(iii) $d_{F}(y) \equiv 0(\bmod 2)$ for all $x \in W$ and $d_{F}(y) \geq 2$ for all $y \in N_{G}\left(u^{*}\right)$.

Clearly, $d_{F}(x) \neq d_{F}(y)$ for all $x y \in E(G)$. We assign weight 1 for each edge of $E(F)$ and weight 0 for each edge of $E(G)-E(F)$. Then we obtain a vertex-coloring $\{0,1\}$-edge-weighting of graph $G$.

Secondly, we show that $G$ admits a vertex-coloring 2-edge-weighting. By Theorem 1.6, we may assume that $G$ is not 3-regular. If $\delta=3$, since $G$ is 3-edge-connected, then $G$ contains a vertex $v^{\prime}$ such that $d_{G}\left(v^{\prime}\right)=3, G-v^{\prime}$ is connected and $\left|N_{G}^{\delta}\left(v^{\prime}\right)\right| \leq 2$. Let

$$
u^{*}= \begin{cases}u & \text { if } \delta \geq 4, \\ v^{\prime} & \text { if } \delta=3 .\end{cases}
$$

Then $u^{*}$ is a vertex satisfying the conditions of Lemma 2.2. Hence by Lemma2.2. $G$ contains a factor $F$ such that 
(i) $d_{F}\left(u^{*}\right)=\delta$

(ii) $d_{F}(x) \equiv \delta(\bmod 2)$ for all $x \in W$ and $d_{F}(x) \leq \delta-2$ for all $x \in N_{G}^{\delta}\left(u^{*}\right)$;

(iii) $d_{F}(y) \equiv \delta+1(\bmod 2)$ for all $y \in U-u^{*}$.

Let $w: E(G) \rightarrow\{1,2\}$ be a 2-edge-weighting such that $w(e)=1$ for each $e \in E(F)$ and $w\left(e^{\prime}\right)=2$ for each $e^{\prime} \in E(G)-E(F)$. Clearly, $c\left(u^{*}\right)=\delta$. If $y \in N_{G}^{\delta}\left(u^{*}\right)$, since there exists an edge $e \sim y$ such that $e \notin E(F)$, then $c(y)=\sum_{e \sim y} w(e)>\delta$. If $y \in N_{G}\left(u^{*}\right)-N_{G}^{\delta}\left(u^{*}\right)$, then $c(y) \geq d_{G}(y)>\delta$. Hence $c(y) \neq c\left(u^{*}\right)$ for all $y \in N_{G}\left(u^{*}\right)$. For each $x y \in E(G)$, where $x \in U-u^{*}$ and $y \in W$, by the choice of $F$, we have $c(x) \equiv \delta+1(\bmod 2)$ and $c(y) \equiv \delta(\bmod 2)$. Hence $w$ is a vertex-coloring $\{1,2\}$-edge-weighting of the graph $G$.

This completes the proof.

Corollary 2.4 Let $G$ be a 3-edge-connected bipartite graph. If $3 \leq \delta(G) \leq 5$, then $G$ admits a vertexcoloring $\mathcal{S}$-edge-weighting for $\mathcal{S} \in\{\{0,1\},\{1,2\}\}$.

Proof: Since $3 \leq \delta \leq 5$ and $G$ is 3-edge-connected, then for every vertex $v$ of degree $\delta, G-v$ is connected. By Lemma 2.2 and Theorem 1.2 , with the same proof, $G$ admits a vertex-coloring $\mathcal{S}$-edgeweighting for $\mathcal{S} \in\{\{0,1\},\{1,2\}\}$.

\section{Conclusions}

In this paper, we prove that every 2-connected and 3-edge-connected bipartite graph admits a vertexcoloring $\mathcal{S}$-edge-weighting for $\mathcal{S} \in\{\{0,1\},\{1,2\}\}$. The generalized $\theta$-graphs is 2 -connected and has a vertex-coloring 3 -edge-weighting but not vertex-coloring $\{0,1\}$-edge-weighting or vertex-coloring 2 edge-weighting. So it is an interesting problem to classify all 2-connected bipartite graphs admitting a vertex-coloring $\mathcal{S}$-edge-weighting. Since the parity-factor problem is polynomial, then there exists a polynomial algorithm to find a vertex-coloring $\mathcal{S}$-edge-weighting of bipartite graphs satisfying the conditions of Theorem 1.2 .

\section{Acknowledgements}

The authors would like to thank the anonymous Reviewer for all valuable comments and suggestions to improve the quality of our paper.

\section{References}

L. Addario-Berry, R. E. L. Aldred, K. Dalal, and B. A. Reed. Vertex coloring edge partitions. J. Combinatorial Theory Ser. B, 94:237-244, 2005.

L. Addario-Berry, K. Dalal, C. McDiarmid, B. A. Reed, and A. Thomason. Vertex-coloring edgeweightings. Combintorica, 27:1-12, 2007.

L. Addario-Berry, K. Dalal, and B. A. Reed. Degree constrained subgraphs. Discrete Applied Math., 156: 1168-1174, 2008.

J. Akiyama and M. Kano. Factors and Factorizations of Graphs - Proof Techniques in Factor Theory. Springer-Verlag Berlin Heidelberg, 2011. 
G. Chang, C. Lu, J. Wu, and Q. Yu. Vertex coloring 2-edge weighting of bipartite graphs. Taiwanese J. Math., 15:1807-1813, 2011.

A. Dudek and D. Wajc. On the complexity of vertex-coloring edgeweightings. Discrete Math. Theor. Comput. Sci., 13:45-50, 2011.

M. Kalkowski, M. Karoński, and F. Pfender. Vertex-coloring edge-weightings: towards the 1-2-3conjecture. J. Combin. Theory Ser. B, 100:347-349, 2010.

M. Karoński, T. Łuczak, and A. Thomason. Edge weights and vertex colors. J. Combin. Theory Ser. B, 91:151-157, 2004.

M. Khatirinejad, R. Naserasr, M. Newman, B. Seamone, and B. Stevens. Vertex-colouring edgeweightings with two edge weights. J. Combin. Theory Ser. B, 14:1-20, 2012.

L. Lovász. The factorization of graphs II. Acta Math. Acad. Sci. Hungar, 23:223-246, 1972.

H. Lu, Q. Yu, and C. Zhang. Vertex-coloring 2-edge-weighting of graphs. European J. Combin., 32: 22-27, 2011.

T. Wang and Q. Yu. A note on vertex-coloring 13-edge-weighting. Frontier Math. in China, 3:1-7, 2008. 
